\title{
EFFECT OF THE COX MAZE PROCEDURE ON THE SECRETION OF ATRIAL NATRIURETIC PEPTIDE
}

Ki-Bong Kim, $\mathrm{MD}^{\mathrm{a}}$

Chang-Ha Lee, MD ${ }^{\mathrm{a}}$

Cheol-Ho Kim, MD ${ }^{\mathrm{a}}$

Young-Joo Cha, $\mathrm{MD}^{\mathrm{b}}$

Sponsor:

James L. Cox, MD
Objectives: The Cox maze procedure has been confirmed to be effective in curing atrial fibrillation. Some authors have reported severe fluid retention after the Cox maze procedure and have suggested decreased secretion of atrial natriuretic peptide as a possible mechanism. This study was designed (1) to examine the serial changes in atrial natriuretic peptide after the Cox maze procedure as compared with changes occurring after coronary artery bypass grafting and (2) to elucidate any differences in atrial natriuretic peptide levels between patients with transient recurrence of atrial fibrillation after the Cox maze procedure and those without recurrence of atrial fibrillation. Methods: Blood samples were drawn from the right and left atria in patients undergoing the Cox maze procedure $(n=19)$ and from the right atrium in patients undergoing coronary artery bypass grafting $(n=6)$ before and 1, 2, and 3 days after the operation. In six patients undergoing the Cox maze procedure, samples were also drawn from the radial artery before and 1, 2, 3, 5, and 7 days after the operation. The plasma samples were prepared by refrigerated centrifugation and stored until radioimmunoassay. In the Cox maze procedure group, atrial natriuretic peptide levels in the right atrium were $629 \pm 366,154 \pm 112,162 \pm 112$, and $183 \pm 97$ $\mathrm{pg} / \mathrm{ml}$ and those in the left atrium were $276 \pm 168,152 \pm 91,162 \pm 111$, and $145 \pm 80 \mathrm{pg} / \mathrm{ml}$ before and 1, 2, and 3 days after the operation, respectively. A marked decrease in atrial natriuretic peptide levels was evident after the Cox maze procedure $(p<0.001)$. There was no significant correlation between atrial natriuretic peptide levels and atrial pressures after the Cox maze procedure, which suggests that secretion of atrial natriuretic peptide by the atria was impaired. There was a significant correlation between the atrial natriuretic peptide levels in the left atrium and those in the peripheral radial artery, and the decreased levels of atrial natriuretic peptide in the radial artery continued for 7 days after the Cox maze procedure. There were no differences in the atrial natriuretic peptide levels between the patients with transient recurrence of atrial fibrillation $(n=6)$ and those without recurrence $(n=13)$ after the Cox maze procedure. In the coronary artery bypass grafting group, the atrial natriuretic peptide levels in the right atrium were $115 \pm 37,124 \pm 48,154 \pm 54$, and $156 \pm 36 \mathrm{pg} / \mathrm{ml}$ before and 1, 2, and 3 days after the operation, respectively. No change was seen after the operation. Conclusions: We observed a significant decrease in atrial natriuretic peptide levels after the Cox maze procedure. This may be
From the Department of Thoracic and Cardiovascular Surgery and Internal Medicine, Seoul National University College of Medicine, ${ }^{\mathrm{a}}$ and Department of Clinical Pathology, Chung Ang University, ${ }^{\text {b Seoul, Korea. }}$

Supported by grant No. 05-95-004 from the Seoul National University Hospital Research Fund.

Read at the Seventy-seventh Annual Meeting of The American Association for Thoracic Surgery, Washington, D.C., May 4-7, 1997.
Received for publication May 7, 1997; revisions requested June 24, 1997; revisions received July 24, 1997; accepted for publication July 25, 1997.

Address for reprints: Ki-Bong Kim, MD, Department of Thoracic and Cardiovascular Surgery, Seoul National University Hospital, 28 Yeunkun-Dong, Chongro-Ku, Seoul 110-744, Korea.

J Thorac Cardiovasc Surg 1998;115:139-47

Copyright (C) 1998 by Mosby, Inc.

$0022-5223 / 98 \$ 5.00+0 \quad \mathbf{1 2} / \mathbf{6} / \mathbf{8 5 0 4 7}$ 
one of the possible causes of fluid retention after this procedure. These decreased atrial natriuretic peptide levels after the Cox maze procedure may result from the multiple atriotomy incisions and excision of both atrial auricles performed during the procedure, rather than from the conversion of atrial fibrillation to normal sinus rhythm. (J Thorac Cardiovasc Surg 1998;115:139-47)

$T_{c}^{\text {he }}$ he Cox maze procedure (CMP), developed by Cox and associates, ${ }^{1,2}$ has become a safe and efficient surgical treatment for medically refractory atrial fibrillation (AF). It has been widely performed as a concomitant procedure in patients with organic cardiac diseases and chronic AF. ${ }^{3,4}$ Despite the beneficial midterm results of the CMP for AF, postoperative fluid retention with pulmonary edema and symptomatic pleural effusion has been observed as one of the early postoperative complications in $12 \%$ to $36 \%$ of the patients. ${ }^{5,6}$ Cox and associates ${ }^{1}$ suggested that this may be the result of a postoperative decrease in the atrial natriuretic peptide (ANP) level that is triggered by the multiple atriotomy incisions and the excision of both atrial auricles that are performed during the CMP. However, few reports have demonstrated a decrease in ANP levels after the CMP.

Several reports have shown a relationship between ANP levels and AF by demonstrating that ANP levels decrease in response to the conversion of AF to normal sinus rhythm. ${ }^{7-9}$ Therefore, there are two possible explanations for the postoperative decrease of ANP levels after the CMP, the surgical incisions that include multiple atriotomies and excision of both atrial auricles and postoperative conversion of AF to normal sinus rhythm. The aims of this study were (1) to examine the serial changes in ANP levels after the CMP and compare them with those after coronary artery bypass grafting (CABG) and (2) to elucidate any differences between ANP levels in patients with transient recurrence of $\mathrm{AF}$ after the CMP and those in patients without recurrence of $\mathrm{AF}$.

\section{Patients and methods}

The CMP was performed in 19 patients with various organic heart diseases combined with AF. Our indications to perform the concomitant CMP were chronic AF, presence of left atrial (LA) thrombus, or medical history of previous thromboembolic events. There were 11 men and 8 women, with a mean age of $46 \pm 8$ years. The mean duration of $\mathrm{AF}$ before the operation was $68 \pm 54$ months (4 to 180 months), and the mean size of the LA on preoperative transthoracic echocardiography was $57 \pm 7$ $\mathrm{mm}$ (37 to $70 \mathrm{~mm}$ ). Most of the patients in the CMP group had been treated before the operation with diuretics and digitalis for congestive heart failure and AF. Eighteen patients, all but the one patient with a ruptured sinus of Valsalva, underwent mitral valve operations. Additional concomitant procedures involved aortic valve operations in nine patients, tricuspid valve surgery in four, closure of atrial septal defect in two, and CABG in one. The CMP III, as described elsewhere by Cox and coworkers, ${ }^{10}$ was performed. After the CMP, six patients $(32 \%)$ had transient recurrence of AF during the early postoperative period. The recurrent AF continued from the operative day until at least the third day after the operation, with conversion to normal sinus rhythm some days later. For comparison with the CMP group, seven patients with ischemic heart disease who were undergoing $\mathrm{CABG}$ were studied. These patients were all male, with a mean age of $54 \pm 8$ years, and were in sinus rhythm before and after the operation.

This study was approved by the hospital ethics committee. Written, informed consent was obtained from each participating patient.

Measurement. In the CMP group, a median sternotomy was performed and the pericardium was opened with the patient under general anesthesia. The pressures were then measured in and blood samples were obtained from both atria. Postoperative blood sampling and pressure measurement of the right atrium (RA) were carried out through a thermodilution catheter, and those of the LA were performed through a pressure line inserted in the right upper pulmonary vein. Postoperative blood sampling and pressure measurements were continued on the first, second, and third postoperative days. Blood samples were also obtained from the radial artery on the first, second, third, fifth, and seventh postoperative days in six cases among the CMP group for comparison with the atrial samples and to examine the serial changes after the operation. In the CABG group, blood samples from the RA and RA pressure measurements were obtained through a thermodilution catheter on the first, second, and third postoperative days.

Specimens of blood from each atrium and the radial artery $(10 \mathrm{ml}$ each $)$ were collected into ethylenediaminetetraacetic acid-treated tubes, stored in ice, and immediately centrifuged at $3000 \mathrm{rpm}$ for 10 minutes at $4^{\circ} \mathrm{C}$ to separate the plasma. The plasma samples were preserved at $-70^{\circ} \mathrm{C}$ until the assay.

ANP concentration was determined by radioimmunoassay with rabbit anti-ANP serum (K-625 Radioimmunoassay kit for Atrial Natriuretic Peptide; Research \& 
Diagnostic Antibodies, Berkeley, Calif.). ANP levels were measured twice for each sample, and a mean was calculated as a representative value.

Statistics. Statistical analysis was performed with the Statistical Analysis System software package (version 6.04; SAS Institute, Cary, N.C.). The significance of changes was assessed by Student's paired $t$ test. Linear regression analysis was used to determine any correlations between the ANP levels in each atrium and independent hemodynamic variables, and also between the ANP levels of both atria and those of the radial artery. Repeated-measures analysis of variance was also performed to investigate the influence of postoperative recurrent $\mathrm{AF}$ on the ANP levels. All results were expressed as mean \pm standard deviation, and a value of $p$ less than 0.05 was considered statistically significant.

\section{Results}

CMP group. The ANP levels in the RA were $629 \pm 366,154 \pm 112,162 \pm 112$, and $183 \pm 97$ $\mathrm{pg} / \mathrm{ml}$ and those in the LA were $276 \pm 168,152 \pm$ 91, $162 \pm 111$, and $145 \pm 80 \mathrm{pg} / \mathrm{ml}$ just after induction of anesthesia and 1,2, and 3 days after the operation, respectively. There was a statistically significant decrease in ANP levels in both atria after the CMP $(p<0.001$; Fig. 1$)$. RA pressures were $8 \pm$ $4,13 \pm 3,15 \pm 3$, and $17 \pm 4 \mathrm{~mm} \mathrm{Hg}$ and LA pressures were $18 \pm 9,17 \pm 4,18 \pm 4$, and $21 \pm 4$ $\mathrm{mm} \mathrm{Hg}$ just after induction of anesthesia and 1, 2, and 3 days after the operation, respectively. The LA pressure was about two times higher than the RA pressure before the operation; however, the preoperative ANP level was about two times higher in the RA than in the LA. There was no significant correlation between the preoperative ANP levels and preoperative pressures in each atrium, or between LA preoperative ANP levels and LA size as measured by preoperative transthoracic echocardiography. Furthermore, there were no significant correlations between the ANP levels and the pressures of each atrium after the CMP.

In the six cases with radial arterial sampling, the ANP levels were $488 \pm 315,255 \pm 107,256 \pm 119$, $220 \pm 75,248 \pm 98$, and $212 \pm 99 \mathrm{pg} / \mathrm{ml}$ just after induction of anesthesia and 1,2,3,5, and 7 days after the operation, respectively (Fig. 2). The decreased ANP levels in the radial artery continued for 7 days after the operation. There was a close correlation between the ANP levels of the LA and those of the radial artery, with correlation coefficients $(r)$ of $0.83(p=0.04), 0.77(p=0.07)$, $0.60(p=0.20)$, and $0.89(p=0.01)$ just after induction of anesthesia and 1,2, and 3 days after the operation, respectively.
There was a statistically significant decrease in the ANP levels both in the patients with transient recurrence of $\mathrm{AF}$ after the operation and in those without recurrence of $\mathrm{AF}$ after the $\mathrm{CMP}(p<$ 0.001). The postoperative ANP levels of the AF recurrence group did not increase significantly despite the $\mathrm{AF}$, and there were no significant differences in the ANP levels between the groups with time (Fig. 3).

CABG group. There were no episodes of AF after the operation in this group, nor were there any significant postoperative changes in RA pressure. The ANP levels in the RA were $115 \pm 37,124 \pm 48$, $154 \pm 54$, and $156 \pm 36 \mathrm{pg} / \mathrm{ml}$ just after induction of anesthesia and 1,2 , and 3 days after the operation, respectively; they showed no significant postoperative change (Fig. 4).

\section{Discussion}

ANP, a 28-amino acid peptide synthesized and secreted by the atria, has several biologic effects. These include natriuresis and diuresis, vasodilation, suppression of renin, and aldosterone secretion. ${ }^{11} \mathrm{~A}$ decrease of ANP level has been suggested as a possible mechanism that causes postoperative fluid retention after the CMP.

This study reveals four main findings. First, the ANP levels in both atria decrease significantly after the CMP, and this may be one of the causes of postoperative fluid retention. Second, there is no significant correlation between the ANP levels and pressures in each atrium after the CMP, suggesting that the secretion of ANP from the atria is impaired. Third, the decreased ANP levels in the radial artery continue until the seventh postoperative day, which suggests that the restoration of atrial mechanical function is delayed at least 1 week after the CMP. Fourth, there are no differences in the ANP levels between patients with transient recurrence of AF after the CMP and those without recurrence; this suggests that the decreased ANP levels after the CMP may result from the multiple atriotomy incisions and excision of both atrial auricles, rather than from the conversion of AF to normal sinus rhythm.

ANP levels at different sites and the relationship between the ANP levels and atrial pressures. Most of our patients had mitral valvular disease, and the LA pressure was about two times higher than the RA pressure before the operation; however, the preoperative ANP levels were about two times higher in the RA than in the LA. A highly significant 


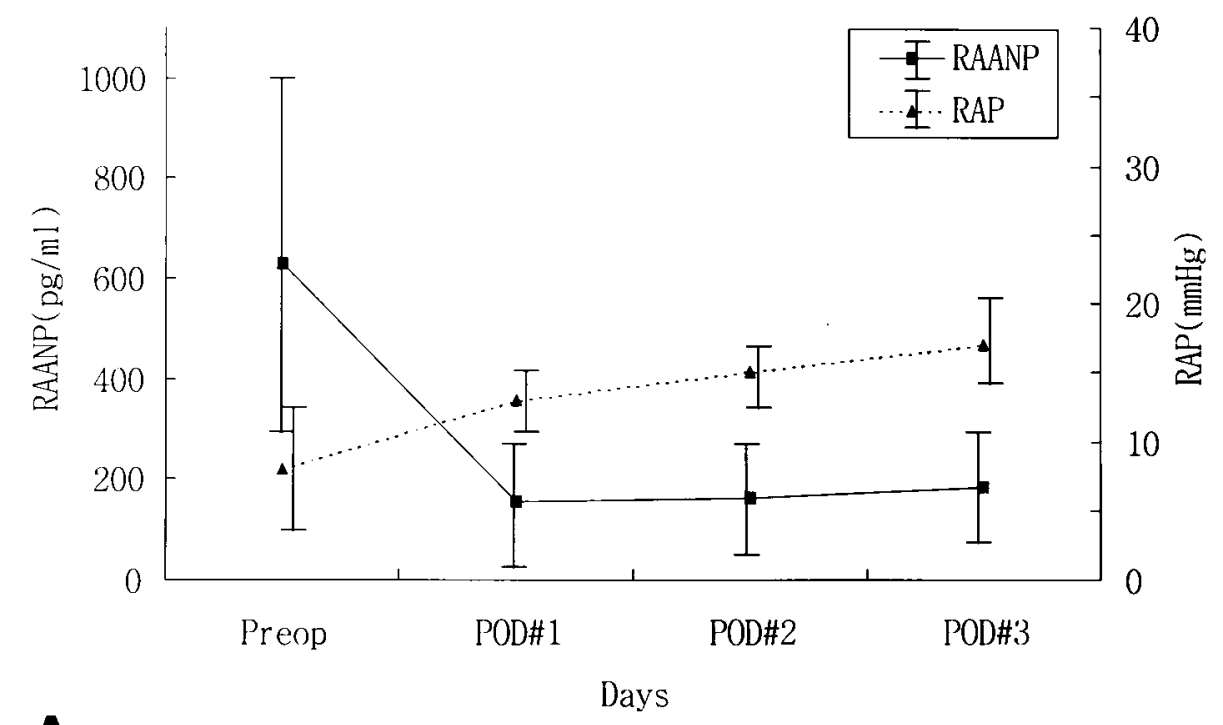

A

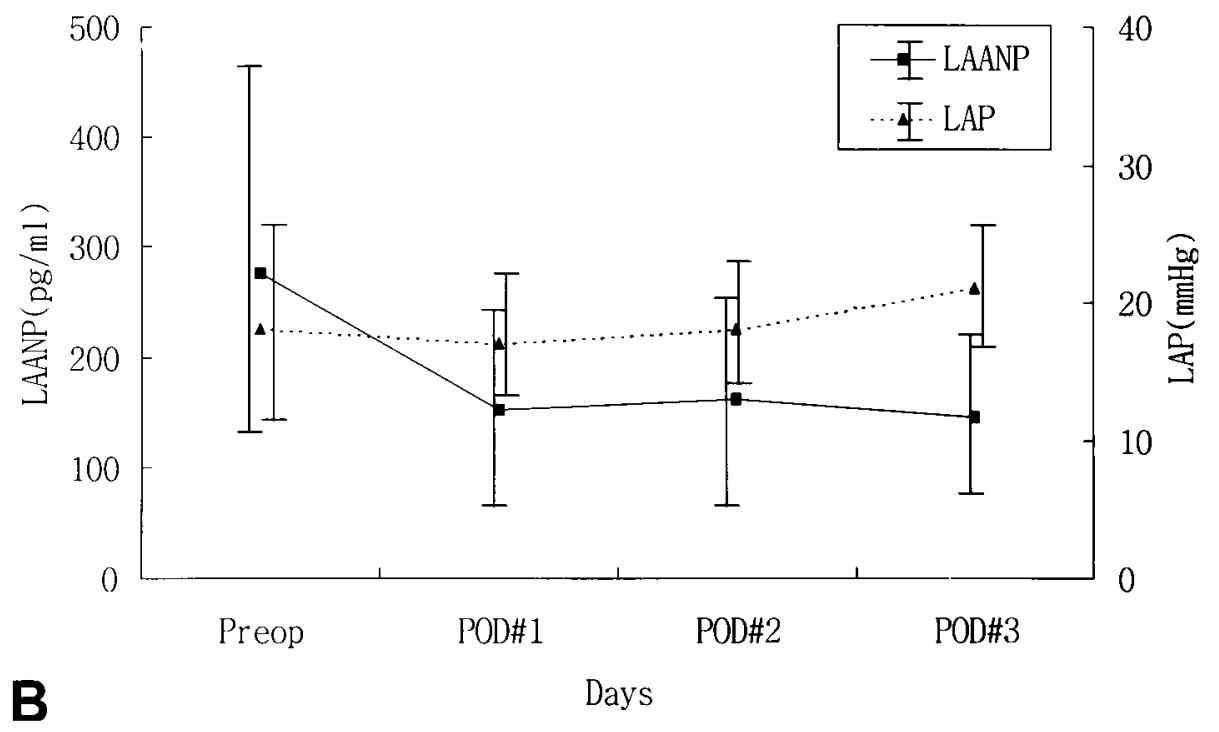

Fig. 1. Changes in atrial ANP levels and pressures in the CMP group. Statistically significant decreases in ANP levels in both RA (A) and LA (B) were seen after the CMP ( $p<0.001)$. RAANP, RA ANP levels; $R A P$, RA pressure; Preop, just after induction of anesthesia; POD, postoperative day; LAANP, LA ANP levels; $L A P$, LA pressure.

increase in ANP levels in the RA was also found in a previous study, reflecting secretion of ANP into the coronary sinus and extraction of ANP by the lungs. ${ }^{12}$ Northridge and associates ${ }^{13}$ showed a higher ANP level in the LA than in the pulmonary veins and demonstrated secretion of the ANP into the LA as well as the RA. Our study demonstrates that there is a significant correlation between the ANP levels of the LA and those of the radial artery, and that the decreased levels of ANP continue until the seventh postoperative day. This close relationship between LA and radial artery levels of ANP 


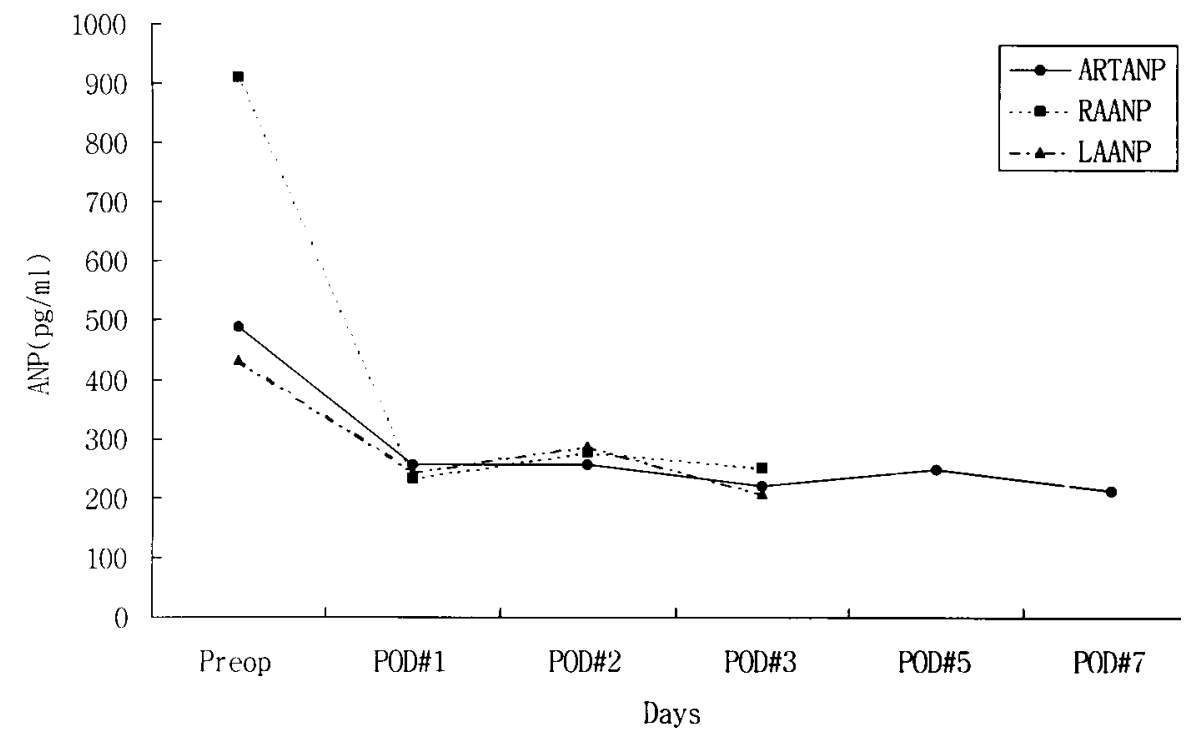

Fig. 2. Changes in the ANP levels of the radial artery (ARTANP) in comparison with those of RA $(R A A N P)$ and LA $(L A A N P)$ in the CMP group. ARTANP showed a close correlation with LAANP, and the decreased ARTANP continued for 7 days after the CMP. Preop, just after induction of anesthesia; $P O D$, postoperative day.

was also found previously. ${ }^{12,13}$ The radial artery ANP levels are therefore indicative of those of the central cardiac chambers.

The search for a mechanism that results in a release of ANP from the atria has produced several demonstrations of a relationship between atrial pressures and plasma ANP levels. ${ }^{7,14,15}$ Another study ${ }^{16}$ emphasized the role of the atrial stretch, rather than atrial pressure, in the release of ANP. Our study shows an insignificant correlation between ANP levels and atrial pressures in the CMP group, suggesting that the secretion of ANP from the atria was impaired.

Postoperative changes in ANP levels. Ashcroft and associates ${ }^{17}$ demonstrated that the postoperative ANP levels in their group of patients undergoing valve replacement showed a smaller increase in postoperative ANP response. They suggested that those findings were the result of a combination of atrial damage and reduced atrial pressure caused by valve replacement or adaptation to chronic fluid overload; thus the valve-replacement group might have been more prone to fluid and salt retention. Our study demonstrates a significant decrease in postoperative ANP levels in comparison with the preoperative ANP levels in both atria of the CMP group. As suggested previously, ${ }^{1}$ this finding may be the result of a postoperative decrease in the ANP levels triggered by the multiple atriotomy incisions and excision of both atrial auricles. In patients with chronic AF, chronically elevated levels of ANP may lead to a resetting of the specific renal receptors ${ }^{7}$; the abrupt postoperative decrease of ANP levels therefore has a high possibility of being the cause of fluid retention. Cox and associates ${ }^{6}$ administered spironolactone to all patients after the operation to prevent postoperative fluid retention, and Perera and associates ${ }^{18}$ suggested the use of ANP after the CMP as a therapeutic possibility. The patients in our study did not have any postoperative fluid retention despite the significant decrease in ANP levels. We believe this is related to preoperative treatment of the patients with diuretics because of some degree of congestive heart failure and postoperative treatment with diuretics to maintain adequate urine output. There were no significant changes in the postoperative ANP levels in the CABG group. Fujiwara and associates ${ }^{9}$ showed a gradual increase in ANP levels in patients with AF from the fourth hour to 5 days after cardioversion, concomitant with an increase in atrial filling fraction. They suggested recovery of atrial mechanical function as the mechanism. In our study, the ANP levels in the radial artery remained decreased until the seventh postop- 


\section{Kim et al.}
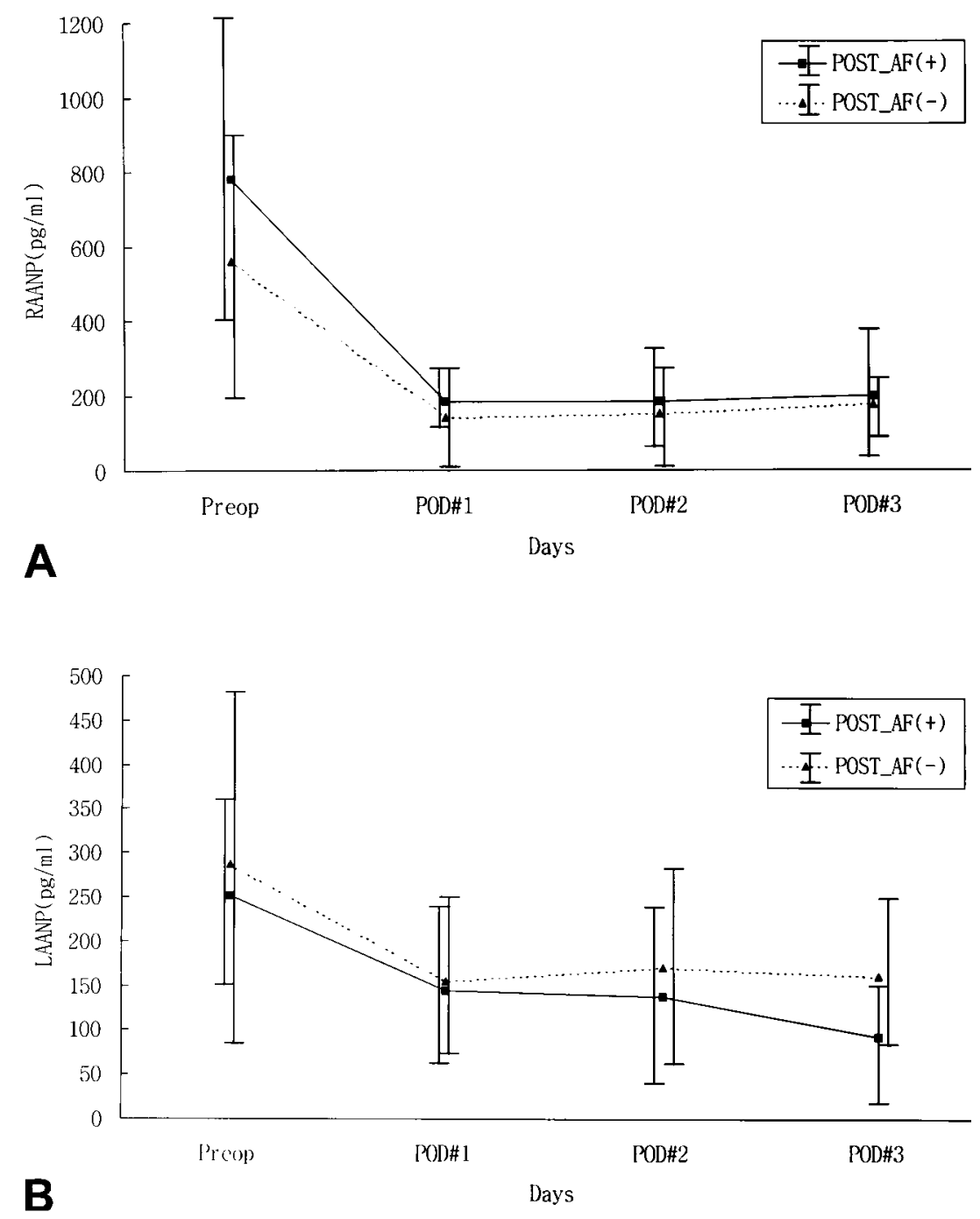

Fig. 3. Comparison of the RA (A) and LA (B) ANP levels between patients with recurrence of AF $(P O S T A F(+))$ and patients without recurrence of AF $(P O S T A F(-))$. There were no significant differences in ANP levels of each atrium between the two groups with time. Preop, just after induction of anesthesia; $P O D$, postoperative day.

erative day. This suggests that atrial mechanical function after the CMP may need more time to recover.

Effect of recurrent AF on ANP levels. Roy and associates $^{7}$ demonstrated a decrease in ANP levels in patients with chronic AF after restoration to sinus rhythm and a significant increase in ANP levels during laboratory-induced supraventricular tachycardia. Dussaule and associates ${ }^{19}$ observed decreases in ANP levels as well as in LA pressures after percutaneous balloon valvuloplasty in pa- tients with mitral stenosis without AF; however, the plasma ANP levels remained elevated in patients with AF despite the decrease in LA pressure. They suggested that the inappropriate response of ANP to the decrease in the LA pressure was caused by the high firing rate of the atria, abnormal stretch of the atrial wall, and larger atrial volume. These observations imply that recovery from the high firing rate of the atria in AF may also be an important factor in the decrease in ANP levels, in addition to the CMP's 


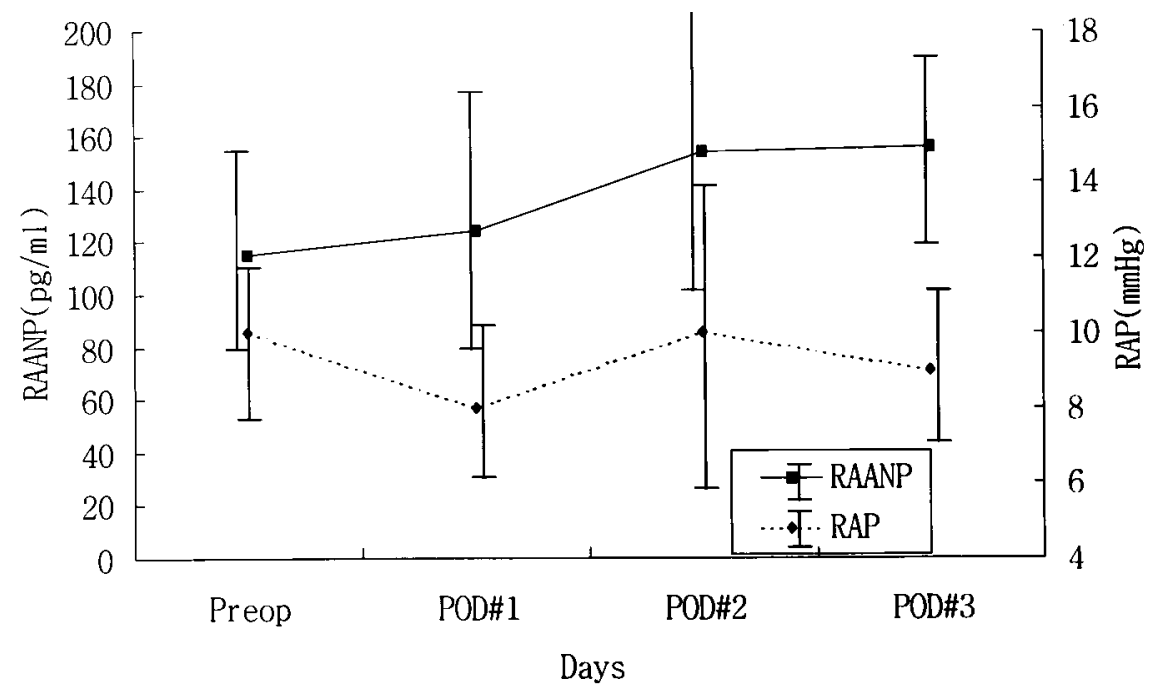

Fig. 4. Changes in the RA ANP levels $(R A A N P)$ and RA pressures $(R A P)$ in the CABG group. There were insignificant changes in the RAANP and RAP after the operation. Preop, just after induction of anesthesia; $P O D$, postoperative day.

multiple atriotomy incisions and excision of both atrial auricles. AF recurred transiently in $32 \%$ of our patients, similar to rates in other reports., 3,6 A comparison of patients with transient postoperative recurrence of AF with those without recurrence showed that postoperative ANP levels in the AF recurrence group did not increase significantly despite the AF, and there was no significant difference in the ANP levels between the two groups with time. Data from our study suggest that decreased ANP levels after the CMP may result from the multiple atriotomy incisions and excision of both atrial auricles, rather than from the conversion of AF to normal sinus rhythm.

Limitations. Several limitations of our study must be recognized. First, there is no accepted world standard for measuring human ANP. ${ }^{20}$ Normal levels of immunoreactive ANP are hard to define, not only because of the lack of assay standardization but also because the condition of subjects in whom normal values are measured varies within and between studied populations. Factors such as the sodium-volume status, systemic blood pressure, posture, age, heart and renal function, and time of sampling all affect the measurement. Second, the perioperative measurement of hemodynamic parameters and blood sampling for the ANP levels were not performed under uniform conditions. Measurements obtained before the operation were performed with patients under general anesthesia, whereas those obtained after the operation were performed with patients in an awake state. This may make it more difficult to interpret the significance of changes in various hemodynamic parameters and ANP levels. Third, the CABG group was used as a control group to compare the postoperative changes in ANP levels. Taking into consideration the multiple factors that may affect ANP levels, a non-CMP group with valvular heart disease and chronic $\mathrm{AF}$ would have been more suitable as a control group, but such a group would have been extremely difficult to incorporate into the study. Fourth, the comparison of patients with transient recurrence of AF with those without recurrence of AF used a small sample size for each group, which may preclude valid statistical comparison of the effect of recurrence of AF on changes in ANP levels.

\section{Conclusions}

Despite several limitations in our study as a clinical approach, we demonstrated a statistically significant decrease in the ANP levels of both atria after the CMP. This may be one of the causes of postoperative fluid retention. This decrease in ANP levels after the CMP may result from the multiple atriotomy incisions and excision of both atrial auricles, rather than from the conversion of AF to normal sinus rhythm. 


\section{REFERENCES}

1. Cox JL, Schuessler RB, D'Agostino HJ Jr, Stone CM, Chang B-C, Cain ME, et al. The surgical treatment of atrial fibrillation. III. Development of a definitive surgical procedure. J Thorac Cardiovasc Surg 1991;101:569-83.

2. Cox JL. The surgical treatment of atrial fibrillation. IV. Surgical technique. J Thorac Cardiovasc Surg 1991;101:58492.

3. McCarthy PM, Cosgrove DM 3rd, Castle LW, White RD, Klein AL. Combined treatment of mitral regurgitation and atrial fibrillation with valvuloplasty and the maze procedure. Am J Cardiol 1993;71:483-6.

4. Kosakai Y, Kawaguchi AT, Isobe F, Sasako Y, Nakano K, Eishi $\mathrm{K}$, et al. Modified maze procedure for patients with atrial fibrillation undergoing simultaneous open heart surgery. Circulation 1995;92(Suppl):II359-64.

5. McCarthy PM, Castle LW, Maloney JD, Trohman RG, Simmons TW, White RD, et al. Initial experience with the maze procedure for atrial fibrillation. $\mathrm{J}$ Thorac Cardiovasc Surg 1993;105:1077-87.

6. Cox JL, Boineau JP, Schuessler RB, Kater KM, Lappas DG. Five-year experience with the maze procedure for atrial fibrillation. Ann Thorac Surg 1993;56:814-24.

7. Roy D, Paillard F, Cassidy D, Bourassa MG, Gutkowska J, Genest J, et al. Atrial natriuretic factor during atrial fibrillation and supraventricular tachycardia. J Am Coll Cardiol 1987;9:509-14.

8. Mookherjee S, Anderson G Jr, Smulyan H, Vardan S. Atrial natriuretic peptide response to cardioversion of atrial flutter and fibrillation and role of associated heart failure. Am J Cardiol 1991;67:377-80.

9. Fujiwara H, Ishikura F, Nagata S, Beppu S, Miyatake K. Plasma atrial natriuretic peptide response to direct current cardioversion of atrial fibrillation in patients with mitral stenosis. J Am Coll Cardiol 1993;22:575-80.

10. Cox JL, Jaquiss RD, Schuessler RB, Boineau JP. Modification of the maze procedure for atrial flutter and atrial fibrillation. II. Surgical technique of the maze III procedure. J Thorac Cardiovasc Surg 1995;110:485-95.

11. Bonow RO. New insights into the cardiac natriuretic peptides. Circulation 1995;93:1946-50.

12. Ashcroft GP, Entwisle SJ, Campbell CJ, Holden MP, Keene ON. Peripheral and intracardiac levels of atrial natriuretic factor during cardiothoracic surgery. Thorac Cardiovasc Surg 1991;39:183-6.

13. Northridge DB, Jamieson MP, Jardine AG, MacArthur KJD, MacFarlane N, Dargie HJ. Pulmonary extraction and left atrial secretion of atrial natriuretic factor during cardiopulmonary bypass surgery. Am Heart J 1992;123:698-703.

14. Raine AEG, Erne P, Buergisser E, Mueller FB, Bolli P, Burkart F, et al. Atrial natriuretic peptide and atrial pressure in patients with congestive heart failure. N Engl J Med 1986;315:533-7.

15. Ledoux S, Dussaule J-C, Michel P-L, Vahanian A, Wolf J-P, Acar J, et al. Acute and delayed hormonal changes in mitral stenosis treated by balloon valvulotomy. Am J Cardiol 1993; 72:932-8

16. Edwards BS, Zimmerman RS, Schwab TR, Heublein DM, Burnett JC Jr. Atrial stretch, not pressure, is the principal determinant controlling the acute release of atrial natriuretic factor. Circ Res 1988;62:191-5.

17. Ashcroft GP, Entwisle SJ, Campbell CJ, Holden MP, Keene
ON. Atrial natriuretic factor release in postoperative cardiothoracic surgical patients. Thorac Cardiovasc Surg 1990;38: 220-3.

18. Perera SA, Frame R, Brodman RF, Zeballos GA, Hintze TH, Panetta TF. Atrial natriuretic peptide replacement therapy in rats subjected to biatrial appendectomy. J Thorac Cardiovasc Surg 1995;109:976-80.

19. Dussaule J-C, Vahanian A, Michel P-L, Soullier I, Czekalski $\mathrm{S}$, Acar J, et al. Plasma atrial natriuretic factor and cyclic GMP in mitral stenosis treated by balloon valvulotomy: effect of atrial fibrillation. Circulation 1988;78:276-85.

20. de Zeeuw D, Janssen WMT, de Jong PE. Atrial natriuretic factor: its (patho)physiological significance in humans. Kidney Int 1992;41:1115-33.

\section{Discussion}

Dr. Tirone E. David (Toronto, Ontario, Canada). Not all patients retain fluid after the CMP. In our experience, which is limited to about 40 cases, I would say that fewer than one in five require long-term diuretic therapy. They do retain fluid for the first 2 or 3 days, but every patient undergoing cardiopulmonary bypass does so. So there must be factors other than the ANP. Do you think that ANP is perhaps just one of several factors? Are there not more factors that may play a role on this problem of fluid retention?

Dr. Kim. Postoperative fluid retention with pulmonary edema has been observed as one of the early postoperative complications after the CMP in $12 \%$ to $36 \%$ of the patients. This incidence is higher than that among the other patients undergoing cardiopulmonary bypass. In our study, we demonstrated a significant decrease in the ANP levels after the CMP. These suggest that the decreased ANP levels after the CMP may play an important role in the problem of further fluid retention. Fortunately, the patients in our study did not have any significant postoperative fluid retention despite the significant decrease in the ANP levels. We believe this is because of preoperative treatment with diuretics in response to some degree of congestive heart failure and postoperative treatment with diuretics to maintain adequate urine output.

Dr. Yoshio Kosakai (Osaka, Japan). We have done the CMP on 270 patients from February 1992 to February 1997. Research similar to yours was performed by our colleague, Dr. Yoshibara. In our results, the ANP value in the CMP group did not increase after the operation. This result was almost the same as yours. However, the ANP value in our control group increased after the operation, because the atrial appendages of the control group were not removed. I think that our result is more reasonable than yours. My question is, what do you think about this difference?

Dr. Kim. Do you mean that the ANP levels in your control group are higher than those in ours?

Dr. Kosakai. Yes, the ANP of our control group increased more after operation than in your results.

Dr. Kim. Did you have the same control group as ours?

Dr. Kosakai. No. All diagnoses in our control group were valvular disease.

Dr. Kim. In our CABG group, the ANP levels in the RA showed no change after the operation. This finding is a little different from those of others, including yours. As 
I indicated in my limitations, there are a couple of possible explanations for this difference. First, there is no accepted world standard for measuring human ANP. Second, a CABG group with relatively small sample size was used as a control group, and this may preclude valid statistical comparison.

Dr. David. Your control group consisted solely of patients undergoing $\mathrm{CABG}$ ?

Dr. Kim. Yes.

Dr. David. You did not have patients undergoing valvular procedures with similar problems?

Dr. Kim. No. Most of our patients undergoing valvular procedures have AF, and I am doing the CMP in all the patients with valvular heart disease with chronic AF, thromboembolic events, or LA thrombus because the CMP can cure chronic AF. So it was extremely difficult to use a non-CMP group with valvular heart disease and chronic $\mathrm{AF}$ as a control group.

Dr. Chi-Ming Wei (Baltimore, Md.). What kind of mechanism do you think decreases ANP? Did you also measure coronary sinus concentrations of ANP and compare aortic position for ANP? In your slides, you show that the decreases in both RA and LA concentrations of ANP differ somewhat from the degree of ANP plasma concentration.

Did you also measure another natriuretic peptide, such as a brain natriuretic peptide? We know that the brain natriuretic peptide is important for the cardiac release of a natriuretic peptide hormone and is also an important contributor to fluid balance.

Dr. Kim. In answer to your first question, we did not measure the ANP levels in coronary sinus or aortic positions. We measured the ANP levels in the RA and the radial artery. The RA preoperative ANP levels were about two times higher than those in the LA or radial artery, reflecting secretion of ANP into the coronary sinus and extraction by the lungs. We suggest that the decreased ANP levels after the CMP may result from the multiple atriotomy incisions and excision of both atrial auricles. In response to the second question, we did not measure the brain natriuretic peptide level.

Dr. Akira T. Kawaguchi (Kanagawa, Japan). I have been wondering about the relationship between decrease in ANP and the denervation of the heart that occurs after the CMP. I also wonder whether you see the ANP level come back up as the heart becomes innervated again. I would like to know the relationship between denervation and innervation of the heart and the level of ANP.

Dr. Kim. Fujiwara and associates, ${ }^{9}$ in the Journal of the American College of Cardiology, showed a gradual increase in the ANP levels in patients with AF from the fourth hour to 5 days after cardioversion, concomitant with an increase in LA filling fraction. In our study, the ANP levels in the radial artery remained decreased until the seventh postoperative day. We are not sure when the ANP levels return to normal. However, if we follow the suggestion of Fujiwara and associates, recovery of ANP secretion after the CMP would be expected to be delayed more than after the cardioversion, because we observed the restoration of the LA function a few months after the operation in most of our patients.

Dr. David. Dr. Cox, would you care to comment on the importance of this hormone in fluid retention. What is the proportion of patients that retain fluid?

Dr. James L. Cox (St. Louis, Mo.). Our results are a bit different, although I have never reported them because I am not sure that I can make any sense out of them. Our actual numbers correlate more with Dr. Kosakai's, in that the ANP levels are elevated on the first postoperative day. By the second or third day after the operation, they are back to control levels. Thereafter they go down, but not as dramatically as in this series.

Having said that, the standard errors of these numbers are similar, in that if you plot each individual result it looks like a scattergram. I think one problem is perhaps in sampling. One of Dr. Kim's initial statements was that there is no worldwide standard for measuring ANP levels, and I believe that this is a key to the confusion of results. Some of the samples are taken from the radial artery, some from the RA, some from the LA, and so on. Dr. Kim is to be congratulated for his efforts to systematizing sampling in the hope of obtaining more meaningful results.

What we do know is that these patients do retain fluid more aggressively after the operation than do patients undergoing $\mathrm{CABG}$ or valvular procedures. They are more comparable with the patients we used to operate on years ago who had severe mitral stenosis and retained a lot of fluid after the operation. Whether the tendency toward excessive fluid retention after the CMP is a problem with ANP, some alteration in the renin-angiotensin system, or a problem with denervation of the heart, I do not know. However, because of this tendency, we started routine use of spironolactone.

I would advise surgeons who are doing this procedure to be aggressive about putting these patients on some regimen to reverse their tendency to retain fluid. I generally leave patients on regimen of spironolactone for 6 weeks and handle additional diuretic needs with furosemide (Lasix). 